\title{
miR-21-5p protects hippocampal neurons of epileptic rats via inhibiting STAT3 expression
}

\author{
${ }^{*}$ Xiaolei Zhang ${ }^{1, A, D, F}$, ${ }^{*}$ Xianfeng $L^{2}{ }^{2, A, C, D, F}$, Bin Li $\mathrm{Li}^{3, B, F}$, Chengfeng Sun ${ }^{4, B, F}$, Peng Zhang ${ }^{5, E, F}$ \\ ${ }^{1}$ Department of Neurology, Jining Combine Traditional Chinese and Western Medicine Hospital, China \\ 2 Department of Neurosurgery, Penglai People's Hospital, Yantai, China \\ ${ }^{3}$ Second Department of Neurology, The Second People's Hospital of Liaocheng, China \\ ${ }^{4}$ Department of Traditional Chinese Medicine, Linyi County Hospital of Traditional Chinese Medicine, Dezhou, China \\ ${ }^{5}$ Department of Neurology, Gaomi People's Hospital, Weifang, China \\ A - research concept and design; $\mathrm{B}$ - collection and/or assembly of data; C - data analysis and interpretation; \\ $D$ - writing the article; $E$ - critical revision of the article; $F$ - final approval of the article
}

\author{
Address for correspondence \\ Peng Zhang \\ E-mail: zhangpeng84kw@163.com

\section{Funding sources} \\ None declared

\section{Conflict of interest \\ None declared \\ * Xiaolei Zhang and Xianfeng Li contributed equally to this work.}

Received on December 1, 2019

Reviewed on January 30, 2020

Accepted on May 1, 2020

Published online on August 3, 2020

Cite as

Zhang X, Li X, Li B, Sun C, Zhang P. miR-21-5p protects hippocampal neurons of epileptic rats via inhibiting STAT3 expression. Adv Clin Exp Med. 2020;29(7):793-801. doi:10.17219/acem/121929

DOI

10.17219/acem/121929

\section{Copyright}

Copyright by Author(s)

This is an article distributed under the terms of the

Creative Commons Attribution 3.0 Unported (CC BY 3.0)

(https://creativecommons.org/licenses/by/3.0/)

\begin{abstract}
Background. Epilepsy is a common chronic neurological disorder worldwide.

Objectives. To investigate the effects of miR-21-5p and signal transducer and activator of transcription-3 (STAT3) expressions on the apoptosis of hippocampal neurons in epileptic rats.

Material and methods. We created a rat model of epilepsy and examined the relationship between miR-21-5p and STAT3 using a bioinformatics website and dual the luciferase reporter (DLR) assay. Real-time quantitative polymerase chain reaction (RT-qPCR) and western blot were used to detect the expression levels of miR-21-5p and STAT3 in hippocampal neurons as well as the protein expression levels of cleaved caspase-3, Bax and BCl-2, which were related to apoptosis of hippocampal neuron. The apoptosis and survival of hippocampal neurons were detected using TUNEL and Nissl staining. Expressions of inflammatory factors interleukin (IL)-6 and tumor necrosis factor a (TNF-a) in serum were examined with enzyme-linked immunosorbent assay (ELISA).
\end{abstract}

Results. miR-21-5p can bind to STAT3. Compared with the miR-21-5p inhibitor negative control (NC) group, the expression levels of caspase-3 and Bax were higher and the expression level of $B C-2$ was lower in the miR-21-5p inhibitor group, whereas the caspase-3 and Bax levels were lower and BCl-2 level was higher in the si-STAT3 (interfering STAT3 gene expression by transfecting small interfering RNA) group (all $p<0.05$ ). Treatment with miR-21-5p inhibitor can lead to significant loss and apoptosis of hippocampal neurons, while interfering with STAT3 expression can reduce the loss and apoptosis of the neurons (all $p<0.05)$. Compared with the miR-21-5p inhibitor NC group, the level of IL-6 was lower in the si-STAT3 group and higher in the miR-21-5p inhibitor group (both $p<0.05$ ).

Conclusions. miR-21-5p can inhibit STAT3 expression and reduce apoptosis and loss of hippocampal neurons and IL-6 level, thereby achieving protective effects on hippocampal neurons of epileptic rats.

Key words: epilepsy, STAT3, hippocampal neuron, miR-21-5p, epileptic rats 


\section{Introduction}

Epilepsy is a common chronic neurological disorder worldwide. It is characterized by recurrent unpredictable seizures and often results from overstimulation of neurons or disorders of metabolism and the nervous system due to brain damage. ${ }^{1-3}$ According to the statistics from the World Health Organization (WHO) on the worldwide prevalence rate of diseases in 2010, epilepsy ranked second among neurological diseases. ${ }^{4}$ The lifespan of patients with epilepsy is much shorter than of healthy people. ${ }^{5}$ Even though the treatment for epilepsy, including medication, neuromodulation, surgical management, and diet control, have been advancing in recent years, nearly $30 \%$ of the patients still do not respond to epilepsy medications and experience regular onset after treatment. ${ }^{6,7}$ Therefore, it is necessary to develop new drugs or gene therapies for better treatment of this disease.

In recent years, the molecular mechanism of gene regulation by microRNA (miRNA) has been gaining attention. MicroRNA can bind to the 3'untranslated region (3'UTR) of its target gene to inhibit mRNA translation. ${ }^{8}$ Some studies have found that miRNA serves a critical role in various biological process, including cell proliferation and growth, inflammation progression, neuronal progenitor synthesis, as well as growth and differentiation of immature neurons. ${ }^{9}$ Of all the discovered miRNAs, about $70 \%$ are specifically expressed in the brain and take a critical part in the function and development of the nervous system. ${ }^{10}$ miR-21-5p, a type of miRNA mainly expressed in brain and neurons, has an essential role in various brain diseases. ${ }^{11-13}$ In the present study, we used bioinformatics website and dual luciferase reporter (DLR) assay to determine whether miR-21-5p can target signal transducer and activator of transcription-3 (STAT3). Other studies have demonstrated that STAT3 overexpression can promote neuronal apoptosis, while inhibiting STAT3 expression can reduce brain damage and neuroinflammation (including the neuroinflammation in patients with cerebral ischemia). ${ }^{14,15}$ Therefore, in the present study, we hypothesized that miR-21-5p could protect the hippocampal neurons of epileptic rats through regulating STAT3 signaling pathway.

\section{Material and methods}

\section{Transfection of miRNA and model creation}

Male Sprague Dawley rats at the age of 6-8 weeks (weight $300 \mathrm{~g}$, purchased from SJA Laboratory Animal, Changsha, China) were kept in separate cages with a constant temperature and humidity. They were fed with a standard diet and sterile water for 1 week and randomized into the following 6 groups with 6 rats in each group: 1) normal group (blank control); 2) model group (epilepsy model); 3) miR-21-5p inhibitor negative control (NC) group (epilepsy model +
miR-21 inhibitor negative control); 4) miR-21-5p inhibitor group (epilepsy model + miR-21-5p inhibition); 5) si-STAT3 group (epilepsy model + STAT3 interference); and 6) miR-21-5p inhibitor + si-STAT3 group (epilepsy model + both miR-21-5p inhibition and STAT3 interference).

All animal experiments followed the institutional guide for the care and use of laboratory animals, and the study was approved by the Ethics Committee of our hospital.

Rat model of epilepsy was induced through an intraperitoneal injection of $127 \mathrm{mg} / \mathrm{kg}$ of lithium chloride, followed by $10 \mathrm{mg} / \mathrm{kg}$ of atropine methobromide $18-24 \mathrm{~h}$ later and another $30 \mathrm{mg} / \mathrm{kg}$ of pilocarpine 30 min later. ${ }^{16}$ Rats in the normal group were intraperitoneally injected with an equal volume of normal saline. The behavior of the rats was observed. According to the Racine's scale, if there was no seizure or the seizure did not reach level IV, intraperitoneal injection of $10 \mathrm{mg} / \mathrm{kg}$ of pilocarpine would be administered every 15 min until status epilepticus (SE) occurred. ${ }^{17}$ Since SE was defined as epilepsy reaching level IV-V and lasting for over $30 \mathrm{~min}$, the SE model was considered to be successfully established if the rats survived and had and epilepsy level over IV. All the reagents used in the model creation were from Sigma-Aldrich (St. Louis, USA).

Lithium chloride-pilocarpine rat model is a common model used in the study on epilepsy. The mechanism of this model creation may be related to the reaction with the acetylcholine receptor. Pilocarpine is an agonist for muscarinic acetylcholine receptors (mAChRs), which can induce a persistent generalized tonic-clonic seizure. The $\mathrm{mAChRs}$ can couple with $\mathrm{G}$ protein, while $\mathrm{G}$ protein can pass the stimulus to phospholipase $C$, which then hydrolyzes phosphatidylinositol 4,5-bisphosphate ( $\left.\mathrm{PIP}_{2}\right)$ to generate two-second messengers, inositol triphosphate (IP3) and diacylglycerol (DG). Excessive levels of IP3 and DG can damage the neuron. Lithium chloride can inhibit the decomposition of IP3, thereby synergizing with pilocarpine.

The sequences of miR-21-5p inhibitor NC, miR-21-5p inhibitor and si-STAT3 (interfering STAT3 gene expression by transfecting small interfering RNA) were synthesized and provided by Ribobio (Guangzhou, China; $5 \mathrm{nmol}$ in each tube). They were diluted with $50 \mu \mathrm{L}$ nuclease-free water to make $5 \mathrm{nmol} / 50 \mu \mathrm{L}$ solution and were divided into 5 tubes of $10 \mu \mathrm{L}$ each, followed by storage at $-20^{\circ} \mathrm{C}$. Before use, the solution of the sequences was diluted to $1 \mathrm{nmol} / 50 \mu \mathrm{L}$ with $40 \mu \mathrm{L}$ pre-cooled normal saline. Five hours after successful modeling, the stereotaxic technique was conducted for administering miR-21-5p inhibitor NC, miR-21-5p inhibitor and si-STAT3. ${ }^{18}$ The rats were fixed onto an animal brain solid positioner after anesthesia. After preoperative skin preparation and disinfection, the skin and muscle were separated to expose the skull bone. The anterior fontanelle was regarded as the origin of coordinate and the right cerebral ventricle was located for drug administration. A microsyringe was inserted for slow administration ( $1 \mathrm{nmol} / 50 \mu \mathrm{L}$ within $30 \mathrm{~min})$ and 
Table 1. RT-qPCR primer sequence

\begin{tabular}{|c|c|c|}
\hline Gene & Forward primer $\left(5^{\prime}-3^{\prime}\right)$ & Reverse primer $\left(5^{\prime}-3^{\prime}\right)$ \\
\hline mir-21-5p & GTCAATAGCTTATCAGACTGA & GTTGGCTCTGGTGCAGGGTCCGAGGTATTCGCA \\
\hline Bax & CTGAGCTGACCTTGGAGC & GACTCCAGCCACAAAGATG \\
\hline $\mathrm{BCl}-2$ & CTGGTGGACAACATCGCTCTG & GGTCTGCTGACCTCACTTGTG \\
\hline Caspase-3 & ATGGACAACAACGAAACCTC & TTAGTGATAAAAGTACAGTTCTT \\
\hline U6 & TGCGGGTGCTCGCTTCGGCAGC & CCAGTGCAGGGTCCGAGGT \\
\hline GADPH & TGGTGAAGGTCGGAGTGAAC & GGAAGATGGTGATGGCCTTTC \\
\hline
\end{tabular}

Bax-BCL2-associated X protein; BCl-2 - B-cell lymphoma-2; GAPDH - glyceraldehyde-3-phosphate dehydrogenase.

then slowly removed, followed by suture and disinfection. Afterward, the rats were normally kept for $48 \mathrm{~h}$.

Sodium pentobarbital ( $30 \mathrm{mg} / \mathrm{kg}, 2 \%$ ) was intraperitoneally injected to the rats for deep anesthesia. The chest was opened and a $50 \mathrm{~mL}$ syringe was inserted into the left cardiac ventricle. The cardiac apex was cut for perfusion with about $150 \mathrm{~mL}$ of normal saline and the blood was removed. Next, a craniotomy was performed to take out the brain tissue, and the hippocampus was located and stored in a labeled freezing tube in liquid-nitrogen for RNA and protein analysis; meanwhile, some samples were fixed with $4 \%$ paraformaldehyde and kept at $4^{\circ} \mathrm{C}$ for subsequent experiments.

\section{Dual-luciferase reporter assay}

We analyzed the pairing of miR-21-5p with STAT3 using a bioinformatics website (www. targetscan.org), followed by DLR assay to verify the targeting of miR-21-tp toward STAT3. The synthesized 3'UTR fragments of STAT3, STAT3-WT (wild) and STAT3-Mut (mutant) were inserted into the downstream of the firefly gene in pMIR-reporter DLR vector (Huayueyang, Beijing, China) to construct recombinant vectors pMIR-STAT3-WT and pMIR-STAT3Mut, respectively. The correctly sequenced DLR plasmid STAT3-WT or STAT3-Mut were then transfected into HEK293T (the Cell Bank of the Chinese Academy of Sciences, Beijing, China) along with miR-21-5p mimic or miR-21-5p NC. The renilla luciferase (Promega, Madison, USA) was used as an internal control, and the cells were collected and lysed after $48 \mathrm{~h}$ of transfection. The firefly luciferase activity and renila luciferase activity were measured with a DLR kit (E1910; Promega) according to the manufacturer's instruction. The value of firefly luciferase activity divided by renilla luciferase activity was calculated for statistical analysis.

\section{RT-qPCR}

The hippocampus tissue was taken out from the liquid nitrogen and weighed. The tissue $(0.1 \mathrm{~g})$ was grounded to fine powder and $1 \mathrm{~mL}$ of Trizol was added (15596026; Invitrogen, Carlsbad, USA). The total RNA was extracted using the single-step method according to the manufacturer's instructions, and the concentration was measured with Eppendorf BioPhotometer (Takara, Kyoto, Japan) before reverse transcription of the total RNAs $(1 \mu \mathrm{g})$ into cDNAs. In accordance with the manufacturer's protocol, the samples were first treated with 5 Xg DNA eraser buffer, gDNA eraser and total RNA for $2 \mathrm{~min}$ at $42^{\circ} \mathrm{C}$ to erase DNA, and then reversely transcribed into cDNAs $\left(37^{\circ} \mathrm{C}\right.$ for $15 \mathrm{~min}$ and $85^{\circ} \mathrm{C}$ for $5 \mathrm{~s}$ ). The real-time quantitative polymerase chain reaction (RT-qPCR) was conducted using SYBR ${ }^{\circledR}$ Premix Ex Taq ${ }^{\mathrm{TM}}$ kit (Tli RNaseH Plus; Takara) and a RT-qPCR device (ABI 7500; Thermo Fisher Scientific, Waltham, USA) with the running parameters were as follows: $95^{\circ} \mathrm{C}$ for $10 \mathrm{~min}$ (pre-denaturation) and 40 PCR cycles (pre-denaturation $95^{\circ} \mathrm{C}$ for $30 \mathrm{~s}$, denaturation $95^{\circ} \mathrm{C}$ for $30 \mathrm{~s}$, annealing for $20 \mathrm{~s}$, and extension $72^{\circ} \mathrm{C}$ for $30 \mathrm{~s}$ ). miR-21-5p was applied as an internal control for $\mathrm{U} 6$, and GAPDH was applied as an internal control for other genes. The primers (GenePharma, Shanghai, China) are listed in Table 1.

\section{Western blot}

The hippocampus tissue was taken from the liquid nitrogen and weighed. The tissue $(0.1 \mathrm{~g})$ was added into an EP tube containing RIPA lysis buffer and protease inhibitor. The homogenate was prepared using an electric pestle. After an ice-bath for $30 \mathrm{~min}$, the samples were centrifuged in order to collect the supernatant, and the protein concentration was measured using a bicinchoninic acid (BCA) kit. Next, the protein samples $(30 \mu \mathrm{g})$ were separated using SDS-PAGE and then transferred to a polyvinyl difluoride (PVDF) membrane (Merck Millipore, Burlington, USA). The membrane was blocked with $5 \%$ bovine serum albumin (BSA) for $90 \mathrm{~min}$ at room temperature, followed by discarding the blocking solution and washing in phosphate-buffered saline with Tween (PBST). Next, the membrane was incubated at $4^{\circ} \mathrm{C}$ overnight with the following antibodies: anti-STAT3 mouse monoclonal antibody (1:1,000, 9139; Cell Signaling Technology, Leiden, the Netherlands), anticleaved caspase-3 rabbit polyclonal antibody (1:1,000, 9664, Cell Signaling Technology), anti-Bax rabbit monoclonal antibody (1:1,000, 14796; Cell Signaling Technology), anti Bcl-2 mouse monoclonal antibody (1:2,000, ab117115; Abcam, Cambridge, UK), and anti-GAPDH rabbit monoclonal antibody (1:10,000, ab181602; Abcam). The membrane was washed in TBST (PBS buffer containing 0.1\% Tween-20) 3 times (10 min per wash) and incubated with horseradish 
peroxidase (HRP)-conjugated goat anti-rabbit and goat antimouse antibody (1:10,000; Jackson ImmunoResearch, West Grove, USA) at room temperature for $1 \mathrm{~h}$. Next, the samples were washed in TBST 3 times (10 min per wash) and then immersed in Pierce enhanced chemiluminescence (ECL) solution (Thermo Fischer Scientific) at room temperature for $1 \mathrm{~min}$. Afterward, the solution was removed and the membrane was covered with plastic wrap and placed in the dark for X-ray film exposure and developing to capture the image. The grayscale of the protein bands was analyzed using ImageJ software (National Institutes of Health, Bethesda, USA) and the results in each experimental group were compared to the internal control for statistical analysis.

\section{TUNEL staining}

The TUNEL staining was conducted using a test kit (MK1020; Boster, Pleasanton, USA). The hippocampus tissue was fixed with $4 \%$ paraformaldehyde and sectioned in $5-\mu \mathrm{m}$ thick slices after paraffin embedding. The samples were dried for $2 \mathrm{~h}$ at $60^{\circ} \mathrm{C}$, followed by dewaxing and hydration with xylene and a gradient of alcohol (concentration from high to low). Subsequently, the samples were incubated with $3 \%$ hydrogen peroxide at room temperature for $10 \mathrm{~min}$ to remove endogenous peroxide and washed with distilled water 3 times. Proteinase K diluted with 0.01 $M$ Tris-buffered saline (TBS) was then added to the sample and digested at $37^{\circ} \mathrm{C}$ for $10 \mathrm{~min}$ followed by 3 washes in $0.01 \mathrm{M}$ TBS. Next, $20 \mu \mathrm{L}$ of labeling buffer was added to keep the section moist $(1 \mu \mathrm{L}$ of terminal deoxynucleotidyl transferase $(\mathrm{TdT})+1 \mu \mathrm{L}$ of digoxigenin with deoxyuridine triphosphate (DIG-d-UTP) $+18 \mu \mathrm{L}$ of labeling buffer for each section). After mixing, the samples were placed in a humidified box for labeling for $2 \mathrm{~h}$ at $37^{\circ} \mathrm{C}$ and washed in $0.01 \mathrm{M}$ TBS 3 times. The samples were then treated with blocking solution for $30 \mathrm{~min}$ at room temperature and with no wash, reacted with anti-digoxigenin biotinylated antibody (1:100, diluted with antibody diluent) at $37^{\circ} \mathrm{C}$ for $30 \mathrm{~min}$ and washed with TBS 3 times, followed by reaction with streptavidin conjugate (SABC) (1:100, diluted with antibody diluent) for $30 \mathrm{~min}$ at $37^{\circ} \mathrm{C}$ and 4 washes in TBS. The samples were then stained with 3,3'diaminobenzidine (DAB), mildly counterstained with hematoxylin, and washed once in TBS and distilled water. Subsequently, the sections were dehydrated, cleared, sealed, and observed under a microscope. Samples taken from each rat had 3 sections and 6 fields were randomly picked in each section. The number of apoptotic cells was calculated using the averaging method. Cell death rate was counted as number of dead neuron/total cell count $\times 100 \%$.

\section{Nissl staining}

The samples were fixed, embedded, dewaxed, and hydrated in the same way as described above. After rinsing with water, $0.1 \%$ of cresyl violet was added for staining at room temperature for $10 \mathrm{~min}$, and the excess liquid was washed away with running water. Dehydration was performed through a gradient of alcohol. The samples were then cleared in dimethylbenzene, mounted with neutral balsam, and observed under a microscope. Live cells were identified as having an intact shape and a purple-blue nucleus. Six fields were randomly picked from each section, and the live neuron and total cell were counted. Cell survival rate was counted as the number of live neuron/ total cell count $\times 100 \%$.

\section{ELISA}

Blood $(0.5 \mathrm{~mL})$ was collected from the tail vein and the serum was separated. The levels of inflammatory factors, interleukin 6 (IL-6) and tumor necrosis factor $\alpha$ (TNF- $\alpha$ ), were measured according to the instructions of the enzyme-linked immunosorbent assay (ELISA) kit (R\&D Systems, Minneapolis, USA).

\section{Statistical analysis}

The SPSS 21.0 v. software (IBM Corp., Armonk, USA) was used for statistical analysis. Measurement data are expressed as mean \pm standard deviation (SD); comparison between 2 groups was conducted with one-way analysis of variance (ANOVA) and Bonferroni post hoc test. P-value $<0.05$ was considered to indicate a statistically significant difference.

\section{Results}

\section{miR-21-5p can target STAT3}

The bioinformatics website TargetScan (www.targetscan.org) predicted that miR-21-5p can target STAT3 (Fig. 1A). The DLR assay was conducted and it was found that, compared with the miR-21-5p inhibitor NC group,

\section{A}

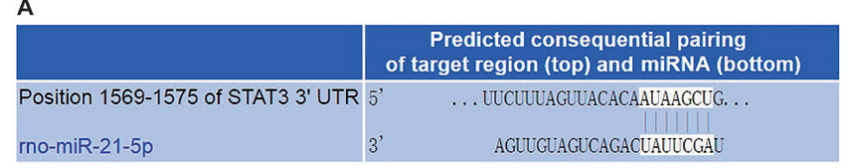

B

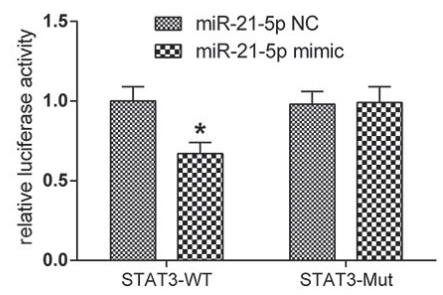

Fig. 1. miR-21-5p can target STAT3. A. The pairing of miR-21-5p and STAT3 as predicted using TargetScan website. B. Dual luciferase reporter verified that miR-21-5p can target STAT3

${ }^{*} p<0.05$ vs the miR-21-5p inhibitor NC group. 
the luciferase activity in the group with co-transfection of miR-21-5p mimic and STAT3-WT was much lower ( $\mathrm{p}<0.05)$, whereas the luciferase activity in the group with co-transfection of STAT3-Mut was similar (Fig. 1B), indicating that miR-21-5p can target STAT3 and regulate its expression.

\section{Expressions of miR-21-5p and STAT3 in rats after transfection}

The RT-qPCR was performed to measure the miR-21-5p expression level in each group (Fig. 2A). The results showed that, compared with the normal group, the expression level of miR-21-5p in the other groups was lower (all $\mathrm{p}<0.05)$. Moreover, the expression levels of miR-21-5p in the miR-21-5p inhibitor and miR-21-5p inhibitor + si-STAT3 groups were lower than those in the model and miR-21-5p inhibitor NC groups (all p < 0.05). No intergroup differences were observed among the model, miR-21-5p inhibitor NC and si-STAT3 in this marker (all p >0.05). The si-STAT3 group had a higher level of miR-21-5p than the miR-21-5p inhibitor and miR-21-5p inhibitor + si-STAT3 groups (both $\mathrm{p}<0.05$ ).

Western blot was performed to detect the protein level of STAT3 in each group (Fig. 2B,C). The results showed that, compared with the normal group, the protein level of STAT3 was higher in the other groups, except for the si-STAT3 group (all p < 0.05). Compared with the model and miR-21-5p inhibitor NC groups, the protein expression level of STAT3 was higher in the miR-21-5p inhibitor group and lower in the si-STAT3 group (both $\mathrm{p}<0.05$ ). The si-STAT3 group had a lower level of STAT3 than the miR-21-5p inhibitor and miR-21-5p inhibitor + si-STAT3 groups (both $\mathrm{p}<0.05)$. No intergroup differences were observed among the model, miR-21-5p inhibitor NC and miR-21-5p inhibitor + si-STAT3 groups in this marker (all p > 0.05).
The results revealed a good transfection rate in each group and showed that suppressing miR-21-5p expression can promote the gene and protein expressions of STAT3, which further verified that miR-21-5p can target STAT3 and negatively regulate its expression.

\section{Protein expressions levels of caspase-3, $B a x$ and $B C l-2$ in hippocampal neurons}

Both RT-qPCR and western blot were performed to measure the expression levels of apoptosis-associated proteins, caspase-3, $B a x$, and $B c l-2$, in hippocampus tissues (Fig. 3). The results displayed that, compared with the normal group, the other groups had higher expression levels of caspase- 3 and $B a x$ and a lower expression level of $\mathrm{Bcl}-2$ (all $\mathrm{p}<0.05)$. Compared with the model and miR-21-5p inhibitor NC groups, the miR-21-5p inhibitor group had higher expression levels of caspase-3 and $B a x$ and a lower expression level of $\mathrm{Bcl}-2$, whereas the si-STAT3 group had lower expression levels of caspase- 3 and $B a x$ and a higher expression level of $B c l-2$ (all $\mathrm{p}<0.05)$. Of the miR-21-5p inhibitor, miR-21-5p inhibitor + si-STAT3 and si-STAT3 groups, the si-STAT3 group had the lowest expression levels of caspase-3 and $B a x$ and the highest level of $B c l$, and the miR-21-5p inhibitor group had the highest expression levels of caspase- 3 and $\mathrm{Bax}$ and the lowest level of $\mathrm{Bcl}$ expression (all p $<0.05$ ). The results demonstrated that apoptosis of hippocampal neurons can be promoted by inhibiting miR-21-5p expression and restrained by interfering with STAT3 expression.

\section{Neuronal apoptosis in rat hippocampus}

The TUNEL staining was conducted to detect the neuronal apoptosis in the CA1 region of rat hippocampus
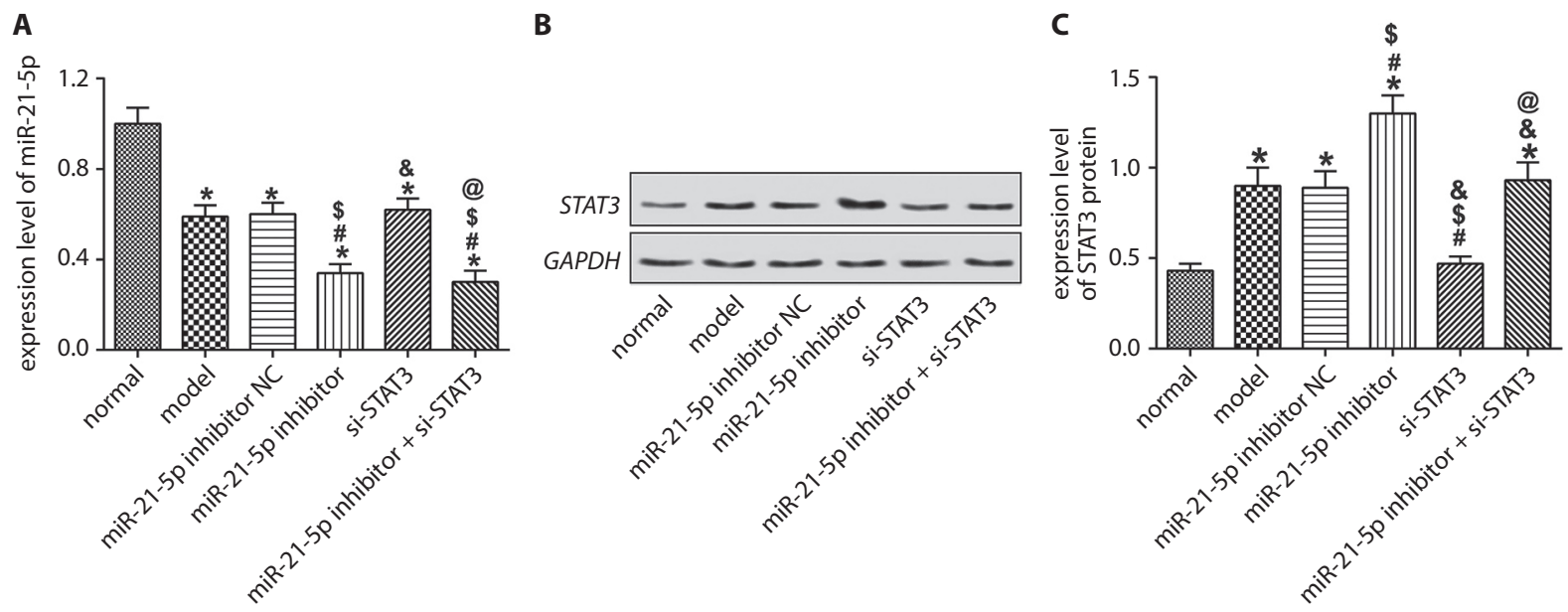

Fig. 2. Expressions of miR-21-5p and STAT3 in epileptic rats. A. miR-21-5p expression level measured using RT-qPCR. B and C. STAT3 expression level measured with western blot and its statistical graph

${ }^{*} p<0.05$ vs the normal group; ${ }^{p} p<0.05$ vs the model group; ${ }^{\$} p<0.05$ vs miR-21-5p inhibitor NC group; ${ }^{\&} p<0.05$ vs miR-21-5p inhibitor group; ${ }^{@} p<0.05$ vs the si-STAT3 group. 
A normal
model
miR-21-5p inhibitor NC
miR-21-5p inhibitor
mi-STAT3
miR-21-5p inhibitor + si-STAT3

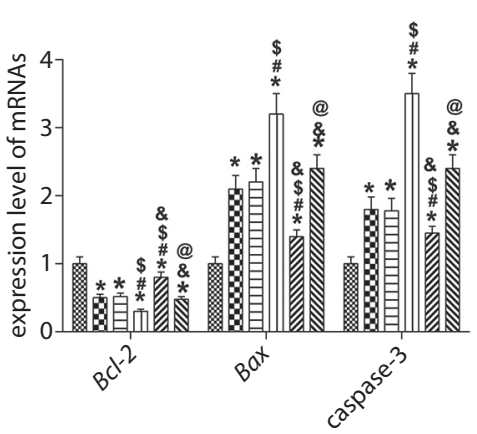

B

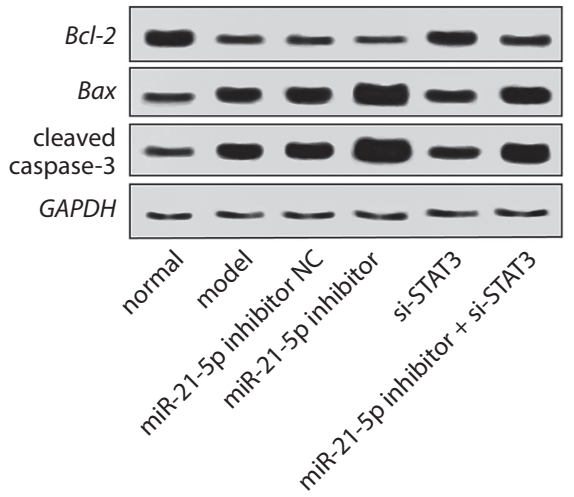

C

$\infty$ model

$\boxminus$ miR-21-5p inhibitor NC

띠 miR-21-5p inhibitor

שIISTAT3

miR-21-5p inhibitor + si-STAT3

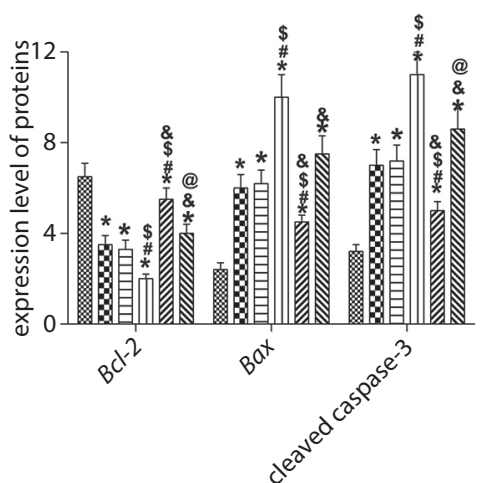

Fig. 3. Expressions of Bcl-2, Bax and capsase-3 in epileptic rats. A. Expression levels of the apoptosis-associated proteins measured with RT-qPCR. B and C. Expression levels of the proteins measured with western blot and its statistical graph

${ }^{*} p<0.05$ vs the normal group; ${ }^{p}<0.05$ vs the model group; ${ }^{\ddagger} p<0.05$ vs miR-21-5p inhibitor NC group; ${ }^{*} p<0.05$ vs miR-21-5p inhibitor group; ${ }^{\circledR} p<0.05$ vs the si-STAT3 group.

A

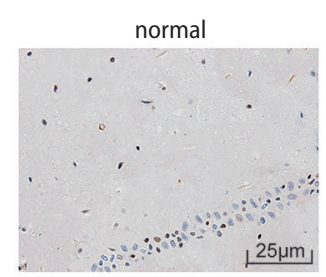

miR-21-5p inhibitor

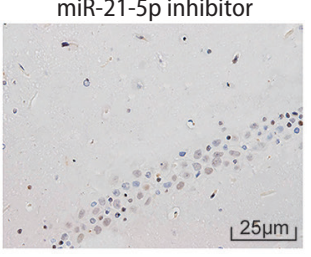

model

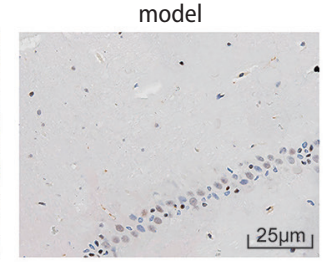

si-STAT3

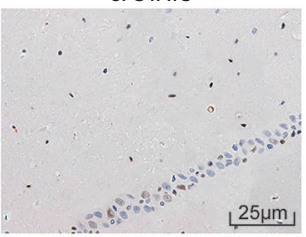

miR-21-5p inhibitor NC

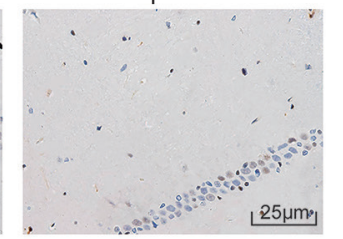

miR-21-5p inhibitor + si-STAT3

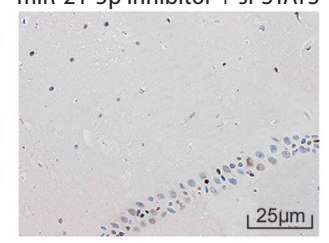

B

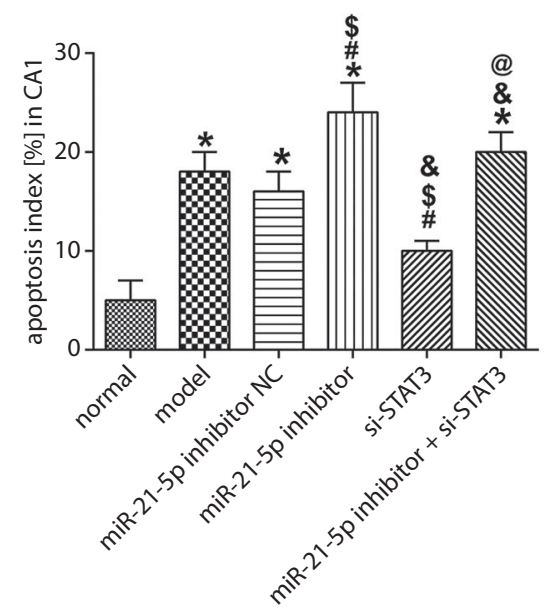

Fig. 4. Neuronal apoptosis in rat hippocampus. A. Neuronal apoptosis in rat hippocampus measured using TUNEL assay. B. Statistical graph of the neuronal apoptosis

${ }^{*} p<0.05$ vs the normal group; $p<0.05$ vs the model group; $\$ p<0.05$ vs miR-21-5p inhibitor NC group; \& $<<0.05$ vs miR-21-5p inhibitor group; ${ }^{\circledR} p<0.05$ vs the si-STAT3 group.

(Fig. 4). The apoptotic cells had an irregular shape, pyknosis and a reduced size of cytoplasm. The results showed that, compared with the normal group, the number of apoptotic cells was higher in the model, miR-21-5p inhibitor NC, miR-21-5p inhibitor, and miR-21-5p inhibitor + si-STAT3 groups (all p < 0.05). Compared with the model and miR-21-5p inhibitor NC groups, the number of apoptotic cells was higher in the miR-21-5p inhibitor group and lower in the si-STAT3 group (both $\mathrm{p}<0.05$ ). Of the miR-21-5p inhibitor, si-STAT3 and miR-21-5p inhibitor + si-STAT3 groups, the number of apoptotic cells was highest in the miR-21-5p inhibitor group and lowest in the si-STAT3 group (all p < 0.05).

\section{Loss and survival of hippocampal neurons}

Nissl staining was performed to detect the loss and survival of hippocampal neurons in the CA1 region in epileptic rats (Fig. 5). Live neurons are those with an intact shape, clear structure, big nucleus, and a high level of Nissl bodies. The results showed that, compared with the normal group, the number of live neurons in the model, miR-21-5p inhibitor NC, miR-21-5p inhibitor, and miR-21-5p inhibitor + si-STAT3 groups was lower (all p $<0.05$ ). Compared with the model and miR-21-5p inhibitor NC groups, the number of live cells was lower in the miR-21-5p inhibitor 
A

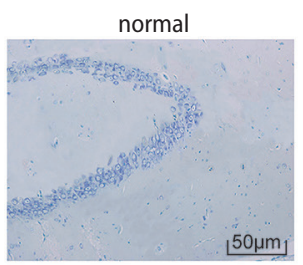

miR-21-5p inhibitor

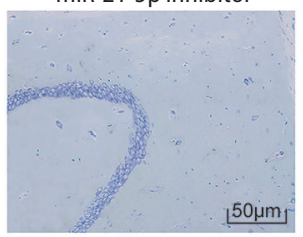

mode

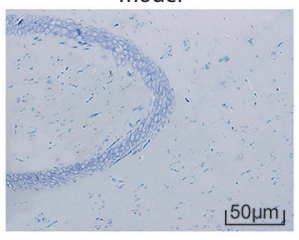

Si-STAT3

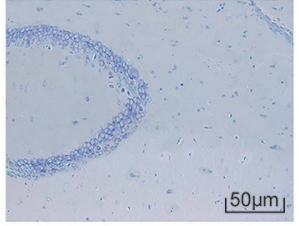

miR-21-5p inhibitor NC

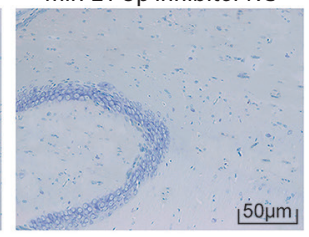

miR-21-5p inhibitor + si-STAT3

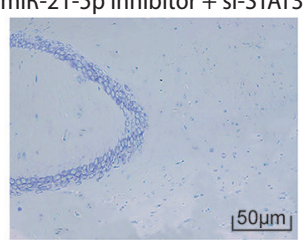

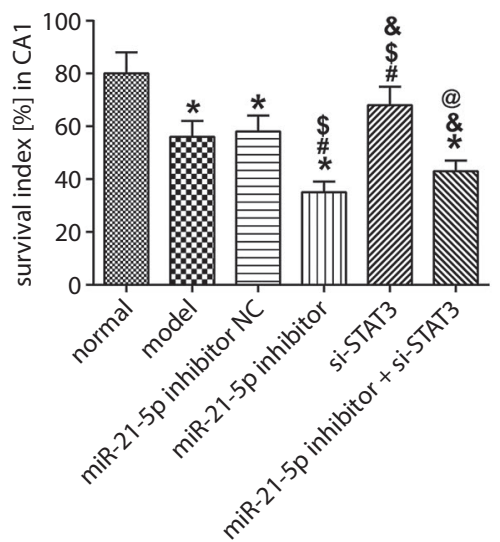

Fig. 5. Survival of hippocampal neurons. A. Survival of hippocampal neurons detected using Nissl staining. B. Statistical graph of the live neurons

${ }^{*} p<0.05$ vs the normal group; $p<0.05$ vs the model group; ${ }^{\$} p<0.05$ vs miR-21-5p inhibitor NC group; ${ }^{\circ} p<0.05$ vs miR-21-5p inhibitor group; ${ }^{\circ} p<0.05$ vs the si-STAT3 group.

group and higher in the si-STAT3 group (all p < 0.05) Of the miR-21-5p inhibitor, si-STAT3, and miR-21-5p inhibitor + si-STAT3 groups, the number of live cells was the lowest in the miR-21-5p inhibitor group and the highest in the si-STAT3 group (all p < 0.05).

\section{Expression levels of IL-6 and TNF-a in serum}

The ELISA was performed to measure the expression levels of inflammatory factors IL- 6 and TNF- $\alpha$ in each group (Fig. 6). The results showed that, compared with the normal group, the IL-6 levels in the other groups were much higher (all $\mathrm{p}<0.05$ ). No intergroup differences were observed among the model, miR-21-5p inhibitor NC and miR-21-5p inhibitor + si-STAT3 groups. Compared with the model and miR-21-5p inhibitor NC groups, the expression level of IL- 6 was higher in the miR-21-5p inhibitor group and lower in the si-STAT3 group (all $p<0.05)$. Of the miR-21-5p inhibitor, si-STAT3 and miR-21-5p inhibitor + si-STAT3 groups, the level of IL- 6 was highest in the miR-21-5p inhibitor group and lowest in the si-STAT3 group (all $\mathrm{p}<0.05)$. Compared with the normal group, the expression level of TNF- $\alpha$ in the other groups was higher (all $\mathrm{p}<0.05)$. Moreover, the expression level of TNF- $\alpha$ was similar among the groups, except for the normal group. These findings showed that miR-21-5p can inhibit STAT3 expression, thus decreasing the expression of IL-6, which suggests that the signal transduction of IL- 6 in epileptic rats requires the participation of STAT3.
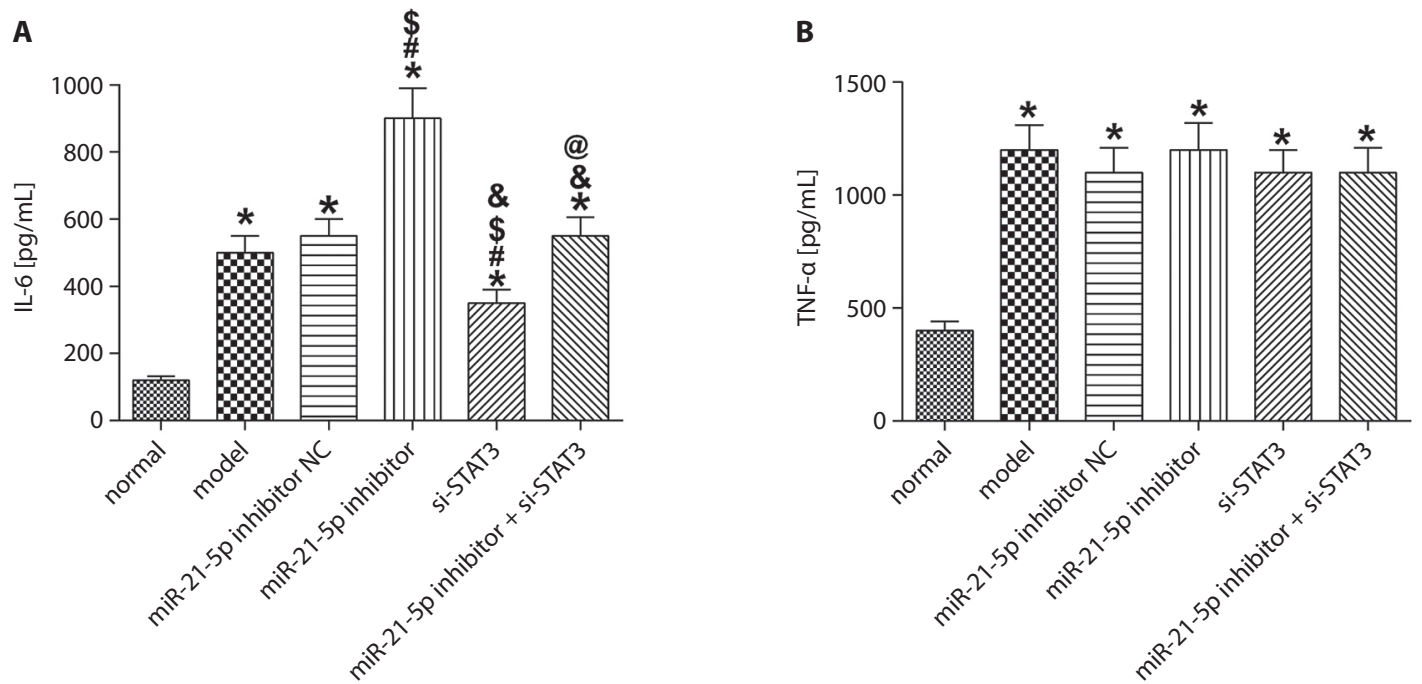

Fig. 6. A. Expression level of IL-6 measured with ELISA. B. Expression level of TNF-a measured with ELISA

${ }^{*} p<0.05$ vs the normal group; ${ }^{*} p<0.05$ vs the model group; ${ }^{\$} p<0.05$ vs miR-21-5p inhibitor NC group; ${ }^{\&} p<0.05$ vs miR-21-5p inhibitor group; ${ }^{@} p<0.05$ vs the si-STAT3 group. 


\section{Discussion}

Our results showed that in the hippocampus of epileptic rats, the expression level of miR-21-5p was low, while the level of STAT3 was high. miR-21 can exert cerebral protective effects in various diseases, alleviate the secondary blood-brain barrier damage after traumatic brain injury in rats, inhibit the neuron death mediated by microglia after hypoxia and ischemia, and prevent hypoxia and low glucose-induced apoptosis of human neural stem cell through suppressing JNK and p38 MAPK signaling pathways. ${ }^{19-23}$ The overexpression of STAT3 gene can lead to neuroinflammation, induce schizophrenia, and is involved in the neurotoxicity caused by arsenic. ${ }^{24-26}$ In our study, we found that miR-21-5p can target STAT3 and regulate its expression after conducting an analysis using a website on bioinformatics and a DLR assay. Compared with the model and miR-21-5p inhibitor NC groups, the miR-21-5p inhibitor group had a higher STAT3 protein expression level, whereas the si-STAT3 group had a lower STAT3 protein expression level, revealing that inhibiting miR-21-5p expression can promote the expression level of STAT3. This finding further demonstrates that miR-21-5p can target STAT3 and negatively regulate its expression.

To further understand the mechanism of miR-21-5p in regulating the gene expression of STAT3 in epilepsy, we measured the levels of apoptosis-associated proteins in rat hippocampus and found that the expression levels of caspase-3 and Bax were higher and expression level of $\mathrm{Bcl}-2$ was lower in the hippocampus of epileptic rats. Moreover, when the miR-21-5p expression was inhibited, the expression levels of caspase- 3 and $B a x$ were further increased and the expression level of $\mathrm{Bcl}-2$ was further reduced, whereas silencing STAT3 reversed these changes. Some studies have demonstrated that miR-21 can suppress apoptosis of many types of cells, including neurons. ${ }^{22,27,28}$ In a study by Shi et al., miR-21 was found to be able to inhibit the protein activity of caspase- 3 and downregulate the ratio of $\mathrm{Bax}$ to $\mathrm{Bcl}-2 .{ }^{29} \mathrm{Caspase}-3$ and $\mathrm{Bax}$ are known to be common apoptosis-associated factors and can promote cell apoptosis when highly expressed. ${ }^{30,31} \mathrm{Bcl}-2$ is an anti-apoptotic protein, which can regulate the permeability of the mitochondrial membrane; however, Bax can damage mitochondrial membrane, thereby promoting cell apoptosis. ${ }^{32}$ Mao et al. have documented that the activation of STAT3 can participate in the induction of neurotoxicity and promote neuronal apoptosis. ${ }^{25}$ Based on these findings and our study results, we concluded that miR-21-5p can reduce the expression of STAT3, thereby inhibiting the neuronal apoptosis in the hippocampus.

Some studies have revealed that neuron activity in the hippocampus can be reduced by a low expression of miR-21-5p and increased by silencing STAT3. Our results showed that in the rat model of epilepsy, the levels of IL- 6 and TNF- $\alpha$ rose significantly. Both IL- 6 and TNF- $\alpha$ are common inflammatory factors, serving critical roles in the development of many diseases. ${ }^{33,34}$ In our study, IL-6 level was increased by silencing miR-21-5p, while TNF- $\alpha$ level remained unchanged after silencing miR-21-5p or inhibiting STAT3 expression. These results showed that miR-21-5p can inhibit STAT3 expression, thus lowering the expression level of IL- 6 , which indicated that the signal transduction of IL-6 in epileptic rats requires the participation of STAT3.

\section{Conclusions}

miR-21-5p can target STAT3 and negatively regulate its expression, increase the neuronal activity in the hippocampus and inhibit the expression of apoptosis-related factors, thereby suppressing neuronal apoptosis. Meanwhile, miR-21-5p can alleviate neuroinflammation in epileptic rats by reducing the inflammatory factor IL- 6 in serum. The understanding of this miRNA may provide some new insights into the treatment of epilepsy. However, the present study only analyzed the mechanism of miR-21-5p in regulating STAT3 and did not investigate the signaling pathways or other molecules that were involved. Therefore, more studies need to be conducted in the future to provide a strong basis for the clinical application of miR-21-5p.

\section{ORCID iDs}

Xiaolei Zhang (D) https://orcid.org/0000-0001-5075-0780 Xianfeng Li (i) https://orcid.org/0000-0002-3364-4051

Bin Li (D) https://orcid.org/0000-0002-0461-7759

Chengfeng Sun (1) https://orcid.org/0000-0001-6540-2164

Peng Zhang (D) https://orcid.org/0000-0003-1937-6860

\section{References}

1. Vezzani A, Fujinami RS, White HS, et al. Infections, inflammation and epilepsy. Acta Neuropathol. 2016;131(2):211-234.

2. Herzberg JL, Fenwick PB. The aetiology of aggression in temporallobe epilepsy. Br J Psychiatry. 1988:153:50-55.

3. Orsini A, Zara F, Striano P. Recent advances in epilepsy genetics. Neurosci Lett. 2018;667:4-9.

4. Zack M, Kobau R. Letter re: Prevalence and incidence of epilepsy: A systematic review and meta-analysis of international studies. Neurology. 2017;89(6):641.

5. Devinsky O, Hesdorffer DC, Thurman DJ, LhatooS, Richerson G. Sudden unexpected death in epilepsy: Epidemiology, mechanisms, and prevention. Neurology. 2016;15(10):1075-1088.

6. Jacobs J, Staba R, Asano E, et al. High-frequency oscillations (HFOs) in clinical epilepsy. Prog Neurobiol. 2012;98(3):302-315.

7. O'Connell BK, Gloss D, Devinsky O. Cannabinoids in treatment-resistant epilepsy: A review. Epilepsy Behav. 2017;70(Pt B):341-348.

8. Shioya M, Obayashi S, Tabunoki H, et al. Aberrant microRNA expression in the brains of neurodegenerative diseases: miR-29a decreased in Alzheimer disease brains targets neurone navigator 3. Neuropathol Appl Neurobiol. 2010;36(4):320-330.

9. Dong RF, Zhang B, Tai LW, Liu HM, Shi FK, Liu NN. The neuroprotective role of miR-124-3p in a 6-hydroxydopamine-induced cell model of Parkinson's disease via the regulation of ANAX5. J Cell Biochem. 2018;119(1):269-277.

10. Sun ZZ, Lv ZY, Tian WJ, Yang Y. MicroRNA-132 protects hippocampal neurons against oxygen-glucose deprivation-induced apoptosis. Int J Immunopathol Pharmacol. 2017;30(3):253-263.

11. Ge X, Huang S, Gao H, et al. miR-21-5p alleviates leakage of injured brain microvascular endothelial barrier in vitro through suppressing inflammation and apoptosis. Brain Res. 2016;1650:31-40. 
12. Yao X, Wang Y, Zhang D. microRNA-21 confers neuroprotection against cerebral ischemia-reperfusion injury and alleviates bloodbrain barrier disruption in rats via the MAPK signaling pathway. J Mol Neurosci. 2018;65(1):43-53.

13. Buller B, Liu X, Wang $X$, et al. MicroRNA-21 protects neurons from ischemic death. FEBS J. 2010;277(20):4299-4307.

14. Wan J, Fu AK, Ip FC, et al. Tyk2/STAT3 signaling mediates beta-amyloid-induced neuronal cell death: Implications in Alzheimer's disease. J Neurosci. 2010;30(20):6873-6881.

15. Hu GQ, Du X, Li YJ, Gao XQ, Chen BQ, Yu L. Inhibition of cerebral ischemia/reperfusion injury-induced apoptosis: Nicotiflorin and JAK2/ STAT3 pathway. Neural Regen Res. 2017;12(1):96-102

16. Curia G, Longo D, Biagini G, Jones RS, Avoli M. The pilocarpine model of temporal lobe epilepsy. J Neurosci Methods. 2008;172(2-4):143-157.

17. Racine RJ. Modification of seizure activity by electrical stimulation. I. After-discharge threshold. Electroencephalogr Clin Neurophysiol. 1972;32(3):269-279.

18. Aronica E, Fluiter K, lyer A, et al. Expression pattern of miR-146a, an inflammation-associated microRNA, in experimental and human temporal lobe epilepsy. Eur J Neurosci. 2010;31(6):1100-1107.

19. Peng J, Omran A, Ashhab MU, et al. Expression patterns of miR-124, miR-134, miR-132, and miR-21 in an immature rat model and children with mesial temporal lobe epilepsy. JMol Neurosci. 2013;50(2):291-297.

20. Zhang H, Wang Y, Lv Q, Gao J, Hu L, He Z. MicroRNA-21 overexpression promotes the neuroprotective efficacy of mesenchymal stem cells for treatment of intracerebral hemorrhage. Front Neurol. 2018;9:931.

21. Ge X, Han Z, Chen F, et al. MiR-21 alleviates secondary blood-brain barrier damage after traumatic brain injury in rats. Brain Res. 2015; 1603:150-157.

22. Zhang L, Dong LY, Li YJ, Hong Z, Wei WS. miR-21 represses FasL in microglia and protects against microglia-mediated neuronal cell death following hypoxia/ischemia. Glia. 2012;60:1888-1895.

23. Chen R, Tai Y, Zhang Y, et al. MicroRNA-21 attenuates oxygen and glucose deprivation induced apoptotic death in human neural stem cells with inhibition of JNK and p38 MAPK signaling. Neurosci Lett. 2019;690:11-16.
24. Wang LC, Liao LX, Zhao MB, Dong X, Zeng KW, Tu PF. Protosappanin A exerts anti-neuroinflammatory effect by inhibiting JAK2-STAT3 pathway in lipopolysaccharide-induced BV2 microglia. Chin J Nat Med. 2017;15(9):674-679.

25. Mao J, Yang J, Zhang Y, et al. Arsenic trioxide mediates HAPI microglia inflammatory response and subsequent neuron apoptosis through p38/JNK MAPK/STAT3 pathway. Toxicol Appl Pharmacol. 2016;303: 79-89.

26. Ma X, Zhou Y, Chai Y, Wang X, Huang X. STAT3 controls maturation and terminal differentiation in mouse hippocampal neurons. $J \mathrm{Mol}$ Neurosci. 2017;61(1):88-95.

27. Han Z, Chen F, Ge X, Tan J, Lei P, Zhang J. miR-21 alleviated apoptosis of cortical neurons through promoting PTEN-Akt signaling pathway in vitro after experimental traumatic brain injury. Brain Res. 2014; 1582:12-20.

28. Zhou S, Zhang S, Wang Y, et al. MiR-21 and miR-222 inhibit apoptosis of adult dorsal root ganglion neurons by repressing TIMP3 following sciatic nerve injury. Neurosci Lett. 2015;586:43-49.

29. Shi L, Chen J, Yang J, Pan T, Zhang S, Wang Z. MiR-21 protected human glioblastoma U87MG cells from chemotherapeutic drug temozolomide induced apoptosis by decreasing $\mathrm{Bax} / \mathrm{Bcl}-2$ ratio and caspase-3 activity. Brain Res. 2010;1352:255-264.

30. Szobi A, Rajtik T, Carnicka S, Ravingerova T, Adameova A. Mitigation of postischemic cardiac contractile dysfunction by CaMKII inhibition: Effects on programmed necrotic and apoptotic cell death. Mol Cell Biochem. 2014;388(1-2):269-276.

31. Fan $Y, L u H, A n L$, et al. Effect of active fraction of Eriocaulon sieboldianum on human leukemia K562 cells via proliferation inhibition, cell cycle arrest and apoptosis induction. Environ Toxicol Pharmacol. 2016;43:13-20.

32. Lv J, Liang Y, Tu Y, Chen J, Xie Y. Hypoxic preconditioning reduces propofol-induced neuroapoptosis via regulation of $\mathrm{Bcl}-2$ and $\mathrm{Bax}$ and downregulation of activated caspase- 3 in the hippocampus of neonatal rats. Neurol Res. 2018;40(9):767-773.

33. Zhang C, Chen Z, Meng X, Li M, Zhang L, Huang A. The involvement and possible mechanism of pro-inflammatory tumor necrosis factor alpha (TNF-alpha) in thoracic ossification of the ligamentum flavum. PLoS One. 2017;12(6):e0178986.

34. Cosenza S, Ruiz M, Maumus M, Jorgensen C, Noel D. Pathogenic or therapeutic extracellular vesicles in rheumatic diseases: Role of mesenchymal stem cell-derived vesicles. Int J Mol Sci. 2017;18(4):889. 